\section{Prevalência do uso de drogas entre escolares do ensino médio do Município de São José do Rio Preto, São Paulo, Brasil}

\author{
Drug abuse prevalence among secondary \\ school students in São José do Rio Preto, \\ São Paulo State, Brazil
}

Elissandro de Freitas Silva 1 Rafael Augusto Borges Pavani 1 Maria Silvia de Moraes 1 Francisco Chiaravalloti Neto 1,2

\footnotetext{
1 Faculdade de Medicina de São José do Rio Preto, São José do Rio Preto, Brasil. 2 Superintendência de Controle de Endemias, São José do Rio Preto, Brasil.

Correspondência R. A. B. Pavani Departamento de Epidemiologia e Saúde Coletiva, Faculdade de Medicina de São José do Rio Preto. Av. Brigadeiro Faria Lima 5416, São José do Rio Preto, SP 15055-430, Brasil. rafael_pavani@universia brasil.net
}

\begin{abstract}
This study investigates the prevalence of drug consumption among secondary school students in São José do Rio Preto, São Paulo State, Southeast Brazil, and its distribution in relation to gender and grade in school. A cross-sectional survey was carried out in São José do Rio Preto. A self-applied questionnaire was answered by a proportional sample of 1,041 teenagers enrolled in $9^{\text {th }}, 10^{\text {th }}$, and $11^{\text {th }}$ grades in public schools. Lifetime consumption of psychoactive substances was: alcohol 77\%, tobacco $28.7 \%$, solvents $18.1 \%$, marijuana $12.1 \%$, amphetamines $3.7 \%$, cocaine $3.3 \%$, hallucinogens $3.1 \%$, and crack $1.4 \%$. Weekly use of marijuana was the highest (2.8\%), followed by solvents (1.3\%). Males consumed more alcohol, marijuana, cocaine, and crack than females. Nighttime use of tobacco, marijuana, cocaine, and hallucinogens was observed. In the present study, prevalence of psychoactive substance use was observed in São José do Rio Preto at rates similar to those found in other Brazilian studies.
\end{abstract}

Street Drugs; Substance-Related Disorders; Students

\section{Introdução}

O abuso de drogas é uma preocupação mundial em função de sua alta freqüência e dos prejuízos sociais, psíquicos e biológicos, com possíveis conseqüências no futuro dos usuários 1,2 . A adolescência constitui uma época de exposição e vulnerabilidade ao uso de drogas 3,4 , em virtude de essa fase ser um período crítico para o desenvolvimento de competências pessoais e interpessoais, aquisição de habilidades para atuar e tomar decisões, tornando-se o abuso de drogas uma forma de lidar com situações problemáticas da vida 4,5 .

É comum a associação entre pobreza, criminalidade e uso de drogas, principalmente pela mídia; contudo, tais relações causais não foram comprovadas em virtude de se tratarem de associações complexas e multilineares, comprovando-se apenas associação entre drogas e violência, especialmente no tráfico de drogas ilegais 6 . A caracterização do consumo de drogas entre escolares torna-se uma importante ferramenta para as políticas públicas na busca de auxílio para a prevenção e tratamento do abuso de drogas lícitas e ilícitas, assim como a procura de fatores associados ao consumo $2,7,8,9,10,11,12,13,14,15,16$.

Muitos estudos encontraram um índice elevado de uso abusivo de drogas lícitas e ilícitas no Brasil 2,7,9,10,11,12,17,18,19, e a fase dos 14 aos 16 anos de idade mostrou o maior índice de jo- 
vens que começam o uso de drogas ilícitas e de tabaco 7,10,12,14. Na adolescência, que inclui essa faixa etária, há rápido desenvolvimento biopsicossocial e a ocorrência de problemas relacionados ao uso de drogas pode influenciar por toda a vida 1,20 .

Levantamentos realizados pelo CEBRID (Centro Brasileiro de Informações sobre Drogas Psicotrópicas) em 1997 entre adolescentes escolares em dez capitais brasileiras mostraram que o uso de maconha e cocaína é maior no sexo masculino 10. Em relação ao uso de drogas em forma de medicamentos, sobretudo os ansiolíticos e anfetamínicos, a prevalência de uso é maior no sexo feminino 10. Comparandose o levantamento de 1987 com o de 1997, percebe-se um aumento significativo do uso de maconha $(2,8 \%$ e $7,6 \%$, respectivamente).

Em Pelotas, Rio Grande do Sul, Brasil, um estudo realizado em 2.410 estudantes do primeiro e segundo graus de escolas públicas e particulares mostrou que o uso de maconha, solventes, cocaína e medicamentos (xaropes, barbitúricos, orexígenos e anticolinérgicos) é maior no sexo masculino. Por outro lado, o sexo feminino apresentou maior prevalência, quando comparado ao sexo masculino, para os ansiolíticos e anfetamínicos 2. Outros estudos nacionais e internacionais, por exemplo no Chile, América Central, México e França obtiveram resultados semelhantes 11,13,14,15,21,22.

O presente estudo tem os objetivos de avaliar a prevalência do consumo de substâncias psicoativas entre escolares do ensino médio do Município de São José do Rio Preto, São Paulo, Brasil, caracterizar a população de estudo e relacionar o consumo com as variáveis sexo e período escolar (diurno e noturno).

\section{Material e método}

\section{Área de estudo, população alvo}

A Cidade de São José do Rio Preto localiza-se na Região Sudeste do Brasil, no Estado de São Paulo, e dista $442 \mathrm{~km}$, em sentido noroeste, da capital. A população estimada para o ano de 2003 era de 382.273 habitantes 23 .

O universo amostral constituiu-se de 425 turmas de 22 escolas públicas de ensino médio com 15.134 alunos matriculados e uma média de 35,6 alunos por turma. Com a Diretoria Regional de Ensino de São José do Rio Preto para cada turma obtiveram-se o número de alunos, o turno (diurno e noturno), a série (primeira, segunda e terceira) e a localização (centro e periferia).

\section{Desenho do estudo e amostragem}

Utilizou-se um estudo de corte transversal em razão de tal abordagem cobrir satisfatoriamente a questão do consumo de substâncias psicoativas e amostragem por conglomerados em único estágio: turmas e alunos 24 .

O cálculo do tamanho da amostra foi realizado considerando-se duas situações: obtenção dos intervalos de confiança e teste de hipóteses. Para o primeiro, consideramos um erro alfa de $5 \%$, uma proporção esperada de $50 \%$, um valor de efeito de desenho máximo de 2,2 e uma precisão de $6 \%$, correspondente a $2,7 \%$ se a amostra fosse casual simples. Para estas condições obtivemos uma amostra de 1.212 alunos.

Em relação à segunda situação calculamos tamanhos de amostras para testes de hipóteses entre sexos e entre turnos (diurno e noturno). No caso da primeira variável fizemos a suposição de que a população estava dividida em 50\% de homens e $50 \%$ de mulheres e que a amostra teria esta mesma distribuição, consideramos um erro alfa de $5 \%$, um poder de $90 \%$, e que para uma proporção esperada de $50 \%$ poderíamos identificar diferenças de $10 \%$. Nessa situação, chegamos a uma amostra de 1.076 alunos. No caso da variável turno, a população era composta por $57 \%$ de alunos do diurno e $43 \%$ do noturno. Para o cálculo do tamanho da amostra fizemos a suposição de que a amostra teria esta mesma distribuição, consideramos um erro alfa de $5 \%$, um poder de $93 \%$, e para uma proporção esperada de $50 \%$ poderíamos identificar diferenças de $10 \%$. Nessa situação chegamos a um tamanho de amostra de 1.202 alunos.

Estabelecemos o tamanho da amostra em 1.212 alunos e, com base em uma taxa de não resposta de $20 \%$ para cobrir possíveis perdas em relação a alunos faltantes no dia da aplicação dos questionários e desistentes, decidimos por aumentar este tamanho para 1.460 alunos. $\mathrm{O}$ número dividido pela média de alunos por turma revelou que deveríamos sortear 41 turmas.

Elaborou-se lista com todas as 425 turmas e as respectivas quantidades de alunos ordenada em primeiro lugar pelo turno, em segundo pela localização e em terceiro pela série. Por intermédio de sorteio sistemático, as 41 turmas foram selecionadas, com a inclusão na amostra de todos os alunos pertencentes a cada uma delas. Três escolas não tiveram salas sorteadas. O sorteio sistemático proporcionou a estratificação da amostra segundo localização, turno e série. 


\section{Instrumento de coleta de dados}

Para a obtenção das informações sobre o consumo de drogas e outros dados, foi utilizado um questionário anônimo auto-aplicado com 66 questões, a maioria pré-codificada, tratando-se de caracterização sócio-demográfica (sexo, estado civil, faixa etária, religião, moradia) e sobre obtenção de conhecimento pelos adolescentes a respeito das drogas. Para dados sobre o uso de drogas, usou-se um instrumento que abordou as seguintes categorias para o uso de substâncias psicoativas, de acordo com a classificação da Organização Mundial da Saúde (OMS) 25:

- Uso na vida: usou pelo menos uma vez na vida;

- Uso no ano: usou pelo menos uma vez nos 12 meses anteriores à pesquisa;

- Uso no mês: usou pelo menos uma vez nos 30 dias anteriores à pesquisa;

- Uso na semana: usou pelo menos uma vez nos 7 dias anteriores à pesquisa;

- Uso freqüente: usou seis vezes ou mais nos 30 dias anteriores à pesquisa;

- Uso pesado: usou 20 vezes ou mais nos 30 dias anteriores à pesquisa.

Realizou-se um pré-teste com a aplicação do questionário em uma turma de alunos e adaptação do material para as dificuldades levantadas. Os questionários foram aplicados de maneira coletiva nas turmas selecionadas e mantidos sem identificação, nos meses de outubro e novembro de 2003. Somente participaram da pesquisa os alunos que estavam presentes na sala de aula no dia da aplicação do questionário, excluindo-se aqueles que não entregaram o Termo de Consentimento Livre e Esclarecido assinado por responsável ou se recusaram a participar da pesquisa.

\section{Análise dos dados}

Os questionários foram conferidos individualmente para a exclusão daqueles entregues em branco ou claramente não fidedignos (discrepâncias entre respostas), e digitados em uma planilha no Microsoft Excel 2000 (Microsoft Corporation, Estados Unidos).

A análise estatística foi realizada nos programas Epi Info 2002 (Centers for Disease Control and Prevention, Atlanta, Estados Unidos) e Stata 7.0 (Stata Corporation, College Station, Estados Unidos) e levou em conta o desenho amostral por conglomerados. Foi considerada como unidade primária de amostragem a turma. Para as proporções, quando referentes ao conjunto de alunos amostrados, calcularam-se os respectivos intervalos de $95 \%$ de confiança.
Foi usado o teste de independência (qui-quadrado) para amostras complexas para comparação das proporções segundo sexo e turno.

\section{Ética da pesquisa}

A pesquisa foi autorizada pelo comitê de ética em pesquisa da Faculdade de Medicina de São José do Rio Preto e cumpre os princípios éticos contidos na Declaração de Helsinki. As escolas sorteadas e seus alunos foram convidados a participar da pesquisa, sem a ocorrência de recusas por parte das escolas. A pesquisa foi autorizada pela Diretoria Regional de Ensino de São José do Rio Preto e pelos diretores de cada escola. Os participantes, ou responsável quando menor, assinaram um termo de consentimento livre e esclarecido para a participação na pesquisa.

\section{Resultados}

Por meio da aplicação dos questionários nas 41 turmas sorteadas, obteve-se uma amostra de 1.035 alunos. As perdas responsáveis pelo não alcance do tamanho de amostra pretendido (1.212 alunos) foram: maior proporção de alunos faltantes e desistentes do valor inicialmente fixado $(20 \%)$; sete recusas em responder o questionário ou preencher o termo de consentimento; e seis questionários desconsiderados, pois apresentavam discrepâncias entre respostas ou estavam em branco.

As características sócio-demográficas estão indicadas na Tabela 1, destacando-se que a maioria dos estudantes estava na faixa etária de 16 a 17 anos (56,6\%), seguida pela faixa de 14 a 15 anos (21,5\%). A parcela mais expressiva (67\%) morava com pai e mãe, e em relação à religião 61,7\% declararam-se católicos. As distribuições por sexo se equivalem com $48,2 \%$ e $51,8 \%$, respectivamente para o sexo masculino e feminino.

A distribuição do consumo de substâncias psicoativas encontra-se na Tabela 2. Em relação ao uso na vida, o álcool é a droga de uso lícito mais consumida, com $77 \%$, e em seguida está o tabaco com $28,7 \%$. Os solventes ocupam o primeiro lugar das substâncias de uso ilícito, com $18,1 \%$, seguidos pela maconha $(12,1 \%)$. Nota-se que as taxas de uso do tabaco manifestam-se inferiores ao álcool em relação a quase todas as categorias exceto para o uso pesado, quando apresentou prevalência mais elevada ( $4,3 \%$ e $1,4 \%$, respectivamente).

Na Tabela 3 é informada a distribuição da prevalência de consumo (na vida) por sexo. Pe- 
Características sócio-demográficas de escolares do ensino médio de escolas públicas. São José do Rio Preto, São Paulo, Brasil, 2003 ( $n=1.035$ ).

\begin{tabular}{|c|c|c|}
\hline Características & $n^{*}$ & $\%$ \\
\hline \multicolumn{3}{|l|}{ Sexo } \\
\hline Masculino & 494 & 48,2 \\
\hline Feminino & 530 & 51,8 \\
\hline \multicolumn{3}{|l|}{ Faixa etária (anos) } \\
\hline $14-15$ & 217 & 21,5 \\
\hline $16-17$ & 572 & 56,6 \\
\hline $18-19$ & 179 & 17,7 \\
\hline 20 ou + & 42 & 4,2 \\
\hline \multicolumn{3}{|l|}{ Estado civil } \\
\hline Solteiro & 992 & 97,3 \\
\hline Outro** & 28 & 2,7 \\
\hline \multicolumn{3}{|l|}{ Série } \\
\hline 10 & 388 & 37,5 \\
\hline 2으 & 325 & 31,4 \\
\hline 3으 & 322 & 31,1 \\
\hline \multicolumn{3}{|l|}{ Período } \\
\hline Diurno & 621 & 60,0 \\
\hline Noturno & 414 & 40,0 \\
\hline \multicolumn{3}{|l|}{ Mora com } \\
\hline Mãe e pai & 683 & 67,0 \\
\hline Só com mãe & 197 & 19,3 \\
\hline Só com pai & 36 & 3,5 \\
\hline Outros ${ }^{\star \star \star}$ & 103 & 10,2 \\
\hline \multicolumn{3}{|l|}{ Religião } \\
\hline Não tem religião & 70 & 6,8 \\
\hline Católica & 633 & 61,7 \\
\hline Evangélica/Protestante & 233 & 22,7 \\
\hline Espírita & 42 & 4,1 \\
\hline Outras\# & 48 & 4,7 \\
\hline
\end{tabular}

* Os totais não coincidem devido à falta de informação para algumas variáveis;

** Outro estado civil: casado; vive com companheiro; *** Cônjuge/companheiro; outros familiares; amigos; sozinho; república; pensão;

\# Afro-brasileira; Testemunha de Jeová.

lo teste qui-quadrado, verificou-se que o consumo de álcool, maconha, cocaína e crack são estatisticamente superiores para o sexo masculino. Os usos na vida de tabaco, solventes e anfetamínicos não apresentaram diferenças significantes quanto ao sexo.

A distribuição da prevalência de consumo (na vida) por período é mostrada na Tabela 4 . Os resultados indicam que existem diferenças estatisticamente significantes para tabaco, maconha, cocaína e alucinógeno, com taxas de prevalência de consumo maiores para o período noturno. O consumo de solvente mostrou prevalência inversa, com o maior consumo sendo superior para o período diurno. Além disso, no período noturno, a maconha passou a ser a droga ilícita mais consumida com $18,6 \%$ de uso na vida, seguida pelos solventes com $17,1 \%$.

\section{Discussão}

O estudo apresenta limitações quanto à generalização dos resultados para todos os alunos de ensino médio do Brasil por tratar de uma localidade específica e de alunos apenas de escolas públicas. A generalização para os adolescentes em geral é ainda mais problemática, visto que entre os adolescentes que não freqüentam a escola a apresentação e a freqüência de consumo de drogas tendem a possuir outro perfil 2,18,21.

Devido à realização da coleta de dados nas classes em dia único e incluir apenas os alunos presentes no momento, o resultado para o uso de drogas pode estar subestimado quanto à freqüência de uso e consumo de drogas mais pesadas, uma vez que os alunos cronicamente faltosos comumente consomem drogas com maior freqüência e utilizam drogas mais pesadas $2,11,18,21$.

No presente estudo, os índices de consumo na vida de álcool e tabaco estão na média brasileira e são inferiores aos índices de Ribeirão Preto, São Paulo 12 e Pelotas, Rio Grande do Sul 2. Quando observamos as taxas para o consumo de maconha, em São José do Rio Preto o uso na vida superou a média brasileira e as Cidades de Ribeirão Preto, Cuiabá, Mato Grosso do Sul e Assis, São Paulo (Tabela 5), assemelhando-se à condição encontrada em Pelotas. O consumo de cocaína, solventes e alucinógenos é geralmente maior em São José do Rio Preto, com exceção para os solventes em Ribeirão Preto e a cocaína em Pelotas e Ribeirão Preto, com taxas respectivamente muito maior e similares. No entanto, quando partimos para o uso no mês, o consumo de solventes praticamente se iguala em São José do Rio Preto (3,4\%) e Ribeirão Preto $(3,3 \%) 12$.

Esses resultados mostram uma situação preocupante em São José do Rio Preto. Apesar de o consumo de drogas lícitas (álcool e tabaco) na vida apresentarem índices semelhantes a outras localidades, ou até inferiores, o consumo de drogas ilícitas em geral é superior ao encontrado em outros estudos. O índice de 12,1\% 
Prevalência do consumo de substâncias psicoativas entre escolares do ensino médio de escolas públicas. São José do Rio Preto, São Paulo, Brasil, 2003.

\begin{tabular}{|c|c|c|c|c|c|c|c|c|c|c|c|c|c|c|}
\hline \multirow[t]{2}{*}{ Substâncias } & \multicolumn{3}{|c|}{ Uso na vida } & \multicolumn{3}{|c|}{ Último ano } & \multicolumn{3}{|c|}{ Último mês } & \multicolumn{3}{|c|}{ Uso freqüente* } & \multicolumn{2}{|c|}{ Uso pesado** } \\
\hline & $N$ & $\%$ & IC95\% & $N$ & $\%$ & IC95\% & $N$ & $\%$ & IC95\% & $\mathrm{N}$ & $\%$ & IC95\% & $\mathrm{N}$ & $\% \quad I C 95 \%$ \\
\hline Álcool & 793/1.030 & 77,0 & $73,5-80,4$ & $660 / 1.029$ & 64,1 & $59,5-68,7$ & $445 / 1.028$ & 43,3 & $38,2-48,3$ & $155 / 1.024$ & 15,1 & $11,9-18,3$ & $14 / 1.024$ & $1,4 \quad 0,5-2,1$ \\
\hline Tabaco & $294 / 1.026$ & 28,7 & $24,9-32,3$ & & $\star \star \star$ & & $114 / 1.023$ & 11,1 & $8,2-14,0$ & $51 / 1.023$ & 4,9 & $3,3-6,5$ & $44 / 1.023$ & $4,3 \quad 2,8-5,7$ \\
\hline Substâncias & Usc & o nav & ida & Últ & imo a & no & & imo $n$ & hês & Uso r & na ser & mana & & \\
\hline Solventes & $185 / 1.022$ & 18,1 & $14,4-21,7$ & $75 / 1.017$ & 7,3 & $5,4-9,2$ & $35 / 1.017$ & 3,4 & $2,1-4,6$ & $13 / 1.017$ & 1,3 & $0,5-1,9$ & & \\
\hline Maconha & $125 / 1.019$ & 12,1 & $8,8-15,5$ & $76 / 1.021$ & 7,4 & $4,9-9,9$ & $42 / 1.021$ & 4,1 & $2,3-5,9$ & 29/1.019 & 2,9 & $1,5-4,3$ & & \\
\hline Anfetamínico & $38 / 1.014$ & 3,7 & $2,2-5,2$ & $23 / 1.019$ & 2,2 & $0,9-3,5$ & 10/1.019 & 1,0 & $0,2-1,7$ & $3 / 1.019$ & 0,3 & $0,0-0,6$ & & \\
\hline Cocaína & $34 / 1.027$ & 3,3 & $1,7-4,9$ & $19 / 1.024$ & 1,9 & $0,9-2,7$ & $7 / 1.027$ & 0,7 & $0,2-1,1$ & & $\star \star \star ~$ & & & \\
\hline Alucinógeno & $32 / 1.018$ & 3,1 & $1,6-4,6$ & $19 / 1.019$ & 1,9 & $0,6-3,0$ & $11 / 1.019$ & 1,1 & $0,3-1,7$ & $2 / 1.019$ & 0,2 & $0,0-0,4$ & & \\
\hline Crack & $14 / 1.028$ & 1,4 & $0,5-2,2$ & $9 / 1.025$ & 0,9 & $0,2-1,5$ & $7 / 1.025$ & 0,7 & $0,1-1,2$ & $4 / 1.025$ & 0,4 & $0,0-0,7$ & & \\
\hline
\end{tabular}

* Uso em 6 ou mais dias no último mês;

** Uso em 20 ou mais dias no último mês;

*** Dados não levantados.

Tabela 3

Prevalência do consumo de substâncias psicoativas (na vida) entre escolares do ensino médio, segundo o sexo. São José do Rio Preto, São Paulo, Brasil, 2003.

\begin{tabular}{lccccccc}
\hline \multirow{2}{*}{ Substâncias } & \multicolumn{2}{c}{ Masculino } & \multicolumn{2}{c}{ Feminino } & \multicolumn{2}{c}{ Total } & \multicolumn{2}{c}{ p valor } \\
& $N$ & $\%$ & $N$ & $\%$ & & $\%$ & \\
\hline Álcool & $400 / 491$ & 81,5 & $383 / 528$ & 72,5 & $783 / 1.019$ & 76,8 & 0,0061 \\
Tabaco & $137 / 490$ & 28,0 & $151 / 526$ & 28,7 & $288 / 1.016$ & 28,3 & 0,7334 \\
Solventes & $91 / 487$ & 18,7 & $92 / 524$ & 17,6 & $183 / 1.011$ & 18,1 & 0,6700 \\
Maconha & $69 / 488$ & 14,1 & $53 / 527$ & 10,1 & $122 / 1.015$ & 12 & 0,0204 \\
Anfetamínico & $23 / 484$ & 4,8 & $14 / 521$ & 2,7 & $37 / 1.005$ & 3,7 & 0,1085 \\
Cocaína & $27 / 489$ & 5,5 & $7 / 527$ & 1,3 & $34 / 1.016$ & 3,3 & 0,0000 \\
Alucinógeno & $18 / 486$ & 3,7 & $13 / 522$ & 2,5 & $31 / 1.008$ & 3,1 & 0,2302 \\
Crack & $11 / 490$ & 2,2 & $3 / 527$ & 0,6 & $14 / 1.017$ & 1,4 & 0,0074 \\
\hline
\end{tabular}

de uso de maconha na vida associado a um uso na semana de 2,8\% (Tabela 2) induz à reflexão dos motivos para esses elevados índices. Essa droga tem histórico de maior aceitação social, facilidade de acesso e por vezes é avaliada como menos prejudicial pelos jovens 26 , mas esses fatos seriam uma explicação mais válida se os estudos demonstrassem índices equivalentes. Como foi observado por Muza et al. 12, as taxas de consumo de maconha foram cerca de duas vezes maiores para os estudos em países desenvolvidos que os estudos na América Latina. Assim, o fato de São José do Rio Preto apresentar privilegiada situação sócio-econômica no Brasil e no Estado de São Paulo, e possuir IDH (Índice de Desenvolvimento Humano) comparável aos países desenvolvidos 27 podem ser explicativos para os resultados encontrados.

Segundo estudos internacionais no Chile 14, América Central 15, Croácia 16,28, México 21, França 22,29, Congo 22 e Noruega 30, além da comparação de Muza et al. 12, o consumo de substâncias psicoativas tende a ser superior nos países desenvolvidos. Na Croácia, Chile e França o consumo de álcool na vida variou entre $74 \% \mathrm{e}$ $90 \% 14,16,22,28$, enquanto na América Central a prevalência média foi de $58 \% 15$. O consumo de tabaco na vida esteve entre $2,5 \%$ no Congo $22 \mathrm{e}$ 
$80 \%$ na Croácia 28 , com prevalência média de $37 \%$ na América Central 15, variando bastante entre os países já citados 14,15,16,22,30. A maconha foi a droga ilícita mais utilizada nos países mais desenvolvidos 14,21,22,28,29,30, com consumo na vida de $16,7 \%$ a cerca de $40 \%$ nos países desenvolvidos, e prevalências menores na América Central, Congo e México 15,21,22. Na América Central o consumo de solventes foi ligeiramente maior que o de maconha $(13 \%$ e $9 \%$, respectivamente) 15. Em São José do Rio Preto o consumo de álcool e maconha assemelhou-se aos países desenvolvidos, enquanto o consumo de tabaco e solventes se parece mais com o encontrado em países em desenvolvimento.

Na distribuição por sexos (Tabela 3), as taxas de consumo na vida de álcool e cocaína foram superiores para o sexo masculino $(\mathrm{p}<0,05)$, como ocorre de forma geral em outros estudos

Tabela 4

Prevalência do consumo de substâncias psicoativas (na vida) entre escolares do ensino médio, segundo o período. São José do Rio Preto, São Paulo, Brasil, 2003.

\begin{tabular}{lccccc}
\hline \multirow{2}{*}{ Substâncias } & \multicolumn{2}{c}{ Diurno } & \multicolumn{2}{c}{ Noturno } & p valor \\
& $N$ & $\%$ & $N$ & $\%$ & \\
\hline Álcool & $469 / 618$ & 75,9 & $324 / 412$ & 78,6 & 0,4208 \\
Tabaco & $147 / 617$ & 23,8 & $147 / 409$ & 35,9 & 0,0010 \\
Solventes & $114 / 609$ & 18,7 & $71 / 413$ & 17,2 & 0,6868 \\
Maconha & $48 / 618$ & 7,8 & $77 / 407$ & 18,9 & 0,0004 \\
Anfetamínico & $16 / 614$ & 2,6 & $22 / 400$ & 5,5 & 0,0519 \\
Cocaína & $9 / 615$ & 1,5 & $25 / 412$ & 6,1 & 0,0016 \\
Alucinógeno & $11 / 611$ & 1,8 & $21 / 407$ & 5,2 & 0,0239 \\
Crack & $5 / 615$ & 0,8 & $9 / 413$ & 2,2 & 0,0905 \\
\hline
\end{tabular}

2,7,11,12,13,14,15,21,31. Souza \& Martins 13 indicaram prevalência similar entre os sexos para a cocaína, e Guimarães et al. 11 e Tavares et al. 2 não encontraram diferença para o álcool. Nesses nove estudos o consumo de solventes foi maior no sexo masculino; porém, no presente trabalho não se encontrou diferença significante $(18,4 \%$ no sexo masculino e $17,4 \%$ no sexo feminino, $\mathrm{p}=0,67$ ).

Com relação ao tabaco, a distribuição por sexos foi idêntica, como também foi observado nos estudos de Tavares et al. 2, Guimarães et al. 11, Souza \& Martins 13 e De Micheli \& Formigoni 19 em Barueri, São Paulo. Segundo Muza et al. 12, em Ribeirão Preto o consumo de tabaco na vida prevaleceu no sexo masculino, assim como em estudos no Chile e América Central 14,15. Na Noruega o consumo de tabaco na vida foi superior no sexo feminino 30 .

De acordo com Tavares et al. 2, Baus et al. 7, Guimarães et al. 11 e Muza et al. 12, encontrou-se consumo de anfetamínicos na vida superior para o sexo feminino, enquanto para Souza \& Martins 13, De Micheli \& Formigoni 19, Dormitzer et al. 15 (América Central) e Rekve \& Lindbaek (Noruega) 30, a distribuição foi semelhante. Em São José do Rio Preto a prevalência foi maior para o sexo masculino (4,8\% contra $2,7 \%$ do sexo feminino) sem diferença significante $(p=0,1085)$. A prevalência para maconha revelou-se maior no sexo masculino ( $14 \%$ contra $10 \%, \mathrm{p}=0,0204$ ), em conformidade com os resultados apresentados no Brasil por Tavares et al. 2, Guimarães et al. 11, Muza et al. 12, Souza \& Martins 13, De Micheli \& Formigoni 19 e Boskovitz et al. 31, e também no exterior 14,15,21,22,30. O consumo de cocaína prevaleceu no sexo masculino nesse estudo e em outros pesquisados 2,11,14,19,21.

Tabela 5

Prevalências de consumo de substâncias psicoativas na vida por localidade*

\begin{tabular}{|c|c|c|c|c|c|c|}
\hline Drogas & $\begin{array}{l}\text { São José do } \\
\text { Rio Preto } \\
(\mathrm{N}=1.035)\end{array}$ & $\begin{array}{c}\text { Brasil } 10 \\
(\mathrm{~N}=15.503)\end{array}$ & $\begin{array}{c}\text { Ribeirão } \\
\text { Preto } 12 \\
(\mathrm{~N}=1.025)\end{array}$ & $\begin{array}{l}\text { Cuiabá } 13 \\
(\mathrm{~N}=1.061)\end{array}$ & $\begin{array}{c}\text { Assis } 11 \\
(N=2.123)\end{array}$ & $\begin{array}{l}\text { Pelotas } 2 \\
(\mathrm{~N}=2.410)\end{array}$ \\
\hline Álcool & 76,6 & 75,9 & 88,9 & 78,6 & 68,9 & 86,8 \\
\hline Tabaco & 28,4 & 32,8 & 37,7 & 29,0 & 22,7 & 41,0 \\
\hline Maconha & 12,1 & 7,6 & 6,2 & 3,8 & 6,6 & 13,9 \\
\hline Solventes & 17,9 & 13,8 & 31,1 & 14,9 & 10,0 & 11,6 \\
\hline Anfetaminas & 3,7 & 4,4 & - & 4,8 & 2,6 & 4,3 \\
\hline Cocaína & 3,3 & 2,0 & 2,7 & 1,8 & 1,6 & 3,2 \\
\hline Alucinógeno & 3,1 & 0,8 & 1,6 & 0,9 & - & - \\
\hline
\end{tabular}

* Os trabalhos incluíram escolares dos ensinos fundamental e médio, exceto o presente estudo. 
O período noturno é freqüentemente associado a um maior consumo de drogas tanto lícitas como ilícitas, como visto em Tavares et al. 2 e De Micheli \& Formigoni 19, e em São José do Rio Preto a apresentação seguiu esse caminho, com maior consumo noturno de tabaco, maconha, cocaína e alucinógeno (Tabela 4). O consumo de solvente, inversamente, apresentou prevalência maior no período diurno, no entanto, sem diferença significante $(p=0,6868)$. Além disso, o consumo noturno de maconha $(18,6 \%)$ superou o consumo de solventes $(17,1 \%)$, tornando-se a droga ilícita mais consumida nesse período. $\mathrm{O}$ álcool não mostrou diferença significante por período.

\section{Conclusões}

O consumo de drogas lícitas e ilícitas relaciona-se com muitos fatores, inclusive com a situação sócio-econômica e cultural de cada lu-

\section{Resumo}

Este estudo dispõe-se a estudar as taxas de prevalência de consumo de substâncias psicoativas entre escolares do ensino médio no Município de São José do Rio Preto, São Paulo, Brasil, e sua distribuição por sexo e período escolar. Utilizou-se um estudo de corte transversal em escolas públicas do ensino médio do município com uma amostragem de conglomerados. Aplicaramse 1.041 questionários autopreenchíveis de maneira coletiva nas classes, mantidos sem identificação. As prevalências do consumo na vida foram: álcool 77\%, tabaco $28,7 \%$, solventes $18,1 \%$, maconha $12,1 \%$, anfetamínicos 3,7\%, cocaína 3,3\%, alucinógenos 3,1\%, $e$ crack $1,4 \%$. O uso na semana de maconha foi o maior (2,8\%), seguido dos solventes (1,3\%). O sexo masculino consumiu mais álcool, maconha, cocaína e crack que o feminino. O período noturno teve prevalência significantemente superior para o tabaco, maconha, cocaína e alucinógeno. No presente estudo, verificou-se uma caracterização da prevalência do consumo de substâncias psicoativas em São José do Rio Preto semelhante à encontrada em outros estudos brasileiros.

Drogas Ilícitas; Transtornos Relacionados ao Uso de Substâncias; Estudantes gar. No presente estudo, verificou-se uma caracterização da prevalência do consumo de substâncias psicoativas em São José do Rio Preto semelhante à encontrada em outros estudos brasileiros e internacionais.

Como o uso de drogas freqüentemente tem seu uso iniciado na adolescência, torna-se importante conhecer a população exposta ao risco do abuso de drogas e agir de forma eficiente. De qualquer forma, a realização de programas para a prevenção do uso de drogas e o tratamento dos usuários deve valorizar os aspectos biopsicossociais do ser humano, geralmente semelhantes em ambientes diversos. Além disso, para o êxito de tais iniciativas é primordial refletir sobre fatores que podem ser protetores para o uso de drogas, tais como bom relacionamento familiar, religiosidade e disponibilidade de informações acerca da dependência e suas conseqüências e perspectivas do futuro.

\section{Colaboradores}

E. F. Silva contribuiu na confecção do projeto, adequação do questionário, aplicação do pré-teste, aplicação dos questionários, digitação dos dados, análise dos dados, redação do artigo (introdução, material e método e resultados). R. A. B. Pavani colaborou na confecção do projeto, adequação do questionário, aplicação do pré-teste, aplicação dos questionários, análise individual dos questionários, análise dos dados, redação do artigo (introdução, discussão e conclusão). M. S. Moraes orientou a pesquisa, participou na aplicação dos questionários, redação do artigo (introdução). F Chiaravalloti Neto contribuiu na análise estatística dos dados, redação do artigo (material e método).

\section{Referências}

1. Colli AS, Saito MI. Adolescência. In: Marcondes E, organizador. Pediatria básica. São Paulo: Editora Sarvier; 1994. p. 539-69.

2. Tavares BF, Béria JU, Lima MS. Prevalência do uso de drogas e desempenho escolar entre adolescentes. Rev Saúde Pública 2001; 35:150-8.

3. Bucher R. Drogas e drogadição no Brasil. Porto Alegre: Artes Médicas; 1992.

4. Rebello S, Monteiro S, Vargas EP. A visão de escolares sobre drogas no uso de um jogo educativo. Interface Comun Saúde Educ 2001; 8:75-88. 
5. Newcomb MD, Bentler PM. Substance use and abuse among children and teenagers. Am Psychol 1989; 44:242-8.

6. Minayo MCS, Deslandes SF. A complexidade das relações entre drogas, álcool e violência. Cad Saúde Pública 1998; 14:35-42.

7. Baus J, Kupek E, Pires M. Prevalência e fatores de risco relacionados ao uso de drogas entre escolares. Rev Saúde Pública 2002; 36:40-6.

8. Carlini-Cotrin B, Barbosa MT. Pesquisas epidemiológicas sobre o uso de drogas entre estudantes: um manual de orientações gerais. São Paulo: Centro Brasileiro de Informações Sobre Drogas Psicotrópicas, Departamento de Psicobiologia, Escola Paulista de Medicina, Universidade Federal de São Paulo; 1993.

9. Carlini EA, Carlini-Cotrin BH, Silva-Filho AR, Barbosa MTS. II Levantamento nacional sobre uso de psicotrópicos em estudantes do 1o e 2o graus. São Paulo: Centro Brasileiro de Informações Sobre Drogas Psicotrópicas, Departamento de Psicobiologia, Escola Paulista de Medicina, Universidade Federal de São Paulo; 1989.

10. Galduróz JCF, Noto AR, Carlini E. IV Levantamento sobre o uso de drogas entre estudantes do 1o e 2o graus em 10 capitais brasileiras. São Paulo: Centro Brasileiro de Informações Sobre Drogas Psicotrópicas, Departamento de Psicobiologia, Escola Paulista de Medicina, Universidade Federal de São Paulo; 1997

11. Guimarães JL, Godinho PH, Cruz R, Kappann JI, Tosta Jr. LA. Consumo de drogas psicoativas por adolescentes escolares de Assis, SP. Rev Saúde Pública 2004; 38:130-2.

12. Muza G, Bettiol H, Muchillo G, Barbieri MA. Consumo de substâncias psicoativas por adolescentes escolares de Ribeirão Preto, SP (Brasil). I - Prevalência do consumo por sexo, idade e tipo de substância. Rev Saúde Pública 1997; 31:21-9.

13. Souza DPO, Martins DTO. O perfil epidemiológico do uso de drogas entre estudantes de 1o e 2o graus da rede estadual de ensino de Cuiabá, Brasil, 1995. Cad Saúde Pública 1998; 14:391-400.

14. Fuentealba R, Cumsille F, Araneda JC, Molina C. Consumo de drogas lícitas e ilícitas en Chile: resultados del estudio de 1998 y comparación con los estudios de 1994 y 1996. Rev Panam Salud Pública 2000; 7:79-87.

15. Dormitzer CM, Gonzalez GB, Penna M, Bejarano J, Obando P, Sanchez M, et al. The PACARDO research project: youthful drug involvement in Central America and the Dominican Republic. Rev Panam Salud Pública 2004; 15:400-16.

16. Jakic M, Jaric-Klinovski Z, Leko V, Jakic M. The incidence of risk behaviours in high school students. Lijec Vjesn 2004; 126:115-20.

17. Carlini EA, Galduróz JC, Noto AR, Nappo AS. I Levantamento Domiciliar Sobre o Uso de Drogas Psicotrópicas no Brasil. São Paulo: Centro Brasileiro de Informações Sobre Drogas Psicotrópi- cas, Departamento de Psicobiologia, Escola Paulista de Medicina, Universidade Federal de São Paulo; 2001.

18. Soldera M, Dalgalarrondo P, Corrêa Filho HR, Silva CAM. Uso de drogas psicotrópicas por estudantes: prevalência e fatores sociais associados. Rev Saúde Pública 2004; 38:277-83.

19. De Micheli D, Formigoni ML. Drug use by Brazilian students: associations with family, psychosocial, health, demographic and behavioral characteristics. Addiction 2004; 99:570-8.

20. D'Andrea FF. Desenvolvimento da personalidade. Rio de Janeiro: Editora Bertrand Brasil; 2001.

21. Medina-Mora ME, Cravioto P, Villatoro J, Fleiz C, Galván-Castillo F, Tapia-Conyer R. Consumo de drogas entre adolescentes: resultados de la Encuesta Nacional de Adicciones, 1998. Salud Pública Mex 2003; 45 Suppl 1:16-25.

22. Courtois R, El-Hage W, Moussiessi T, Mullet E. Prevalence of alcohol, drug use and psychoactive substance consumption in samples of French and Congolese high school students. Trop Doct 2004; 34:15-7.

23. Departamento de Informação e Informática do SUS. Informações demográficas. http://www. tabnet.datasus.gov.br/cgi/tabcgi (acessado em 10/Nov/2004).

24. Kish LS. Survey sampling. Washigton: John Wiley \& Sons; 1967.

25. World Health Organization. Nomenclature and classification of drug and alcohol - related problems: a WHO memorandum. Bull World Health Organ 1981; 59:225-45.

26. Tiba I. A maconha e o jovem - família, escola e sociedade. São Paulo: Editora Agora; 1989.

27. Programa das Nações Unidas para o Desenvolvimento. Atlas do desenvolvimento humano no Brasil, 2003. http://www.pnud.org.br/atlas (acessado em 28/Ago/2004).

28. Ljubotina D, Galic J, Jukic V. Prevalence and risk factors of substance use among urban adolescents: questionnaire study. Croat Med J 2004; 45:88-98.

29. Arditti J, Spadari M, Camprasse MA, Dalecky C, Bourdon JH. Abuse of licit and illicit psychoactive substances in children and teenagers in the PACA Region (Southeastern France). Therapie 2004; 59:595-7.

30. Rekve R, Lindbaek M. Drug abuse among high school students in Hamar in 1999. Tidsskr Nor Laegeforen 2002; 122:2448-51.

31. Boskovits EP, Cruz ETN, Chiaravalloti Neto F, Moraes MS, Paiva Netto JV, Ávila LA, et al. Uso de Drogas entre Estudantes Universitários em São José do Rio Preto, São Paulo. Rev Psiquiatr Clín (São Paulo) 1995; 22:87-93.

Recebido em 06/Dez/2004

Versão final reapresentada em 07/Jun/2005

Aprovado em 21/Out/2005 